\title{
NON-CONVEX CONSENSUS ADMM FOR SATELLITE PRECODER DESIGN
}

\author{
Miguel Ángel Vázquez $z^{\star}$, Aritra Konar ${ }^{\dagger}$, Luis Blanco $^{\star}$, Nicholas D. Sidiropoulos ${ }^{\dagger}$, Ana I. Pérez-Neira ${ }^{\star \dagger \dagger}$ \\ ${ }^{\star}$ Centre Tecnològic de Telecomunicacions de Catalunya (CTTC) \\ $\dagger$ Dept. of Electrical and Computer Engineering University of Minnesota \\ ${ }^{\dagger \dagger}$ Dept. of Signal Theory and Communications Universitat Politècnica de Catalunya
}

\begin{abstract}
Owing to the rapidly increasing traffic demands on satellite connectivity, the current exclusive frequency allocation is becoming obsolete. Instead, aggressive frequency reuse and interference mitigation techniques are promising ideas that both industry and academia are investigating. This paper proposes an optimization precoding technique for dealing with the multibeam interference due to the aggressive frequency reuse. In contrast to general multiuser multiple input multiple output (MIMO) schemes, multibeam satellite precoding techniques call for frame-by-frame quadratically constrained quadratic optimization of a large number of variables. We focus on the multigroup multicast beamforming optimization problem, and we propose to adopt a consensus-based alternating direction method of multipliers (C-ADMM) approach, in order to mitigate complexity. The proposed C-ADMM approach is shown to exhibit comparable optimization performance at considerably lower complexity relative to the prior state-of-art for the formulation considered.
\end{abstract}

Index Terms - Multibeam satellite systems, Precoding, Nonconvex QCQP, ADMM.

\section{INTRODUCTION}

The use of multibeam satellite systems is becoming attractive for mobile operators as a cost-effective solution for providing broadband connectivity everywhere. This is the case of Viasat 1, Echostar 17 and KaSat satellites which employ dozens of beams for providing internet access over a continental coverage area. Due to the forthcoming ubiquitous high data rate demands, both academia and industry are investigating novel schemes in order to increase the overall multibeam satellite capacity.

A very promising approach is the use of full frequency reuse among beams jointly with precoding in order to reduce the multibeam interference [1]. As a result, the multibeam satellite system can switch from the current frequency reuse factor 4 to full frequency reuse, leading to a substantial increase of the user bandwidth. As long as the precoding is able to keep a sufficiently large signal-tonoise ratio (SINR) for each user, the multibeam system capacity will be increased.

Multibeam satellite precoding can be categorized as the wellknown multiuser multiple-input-single-output (MISO) multigroup multicast problem [2]. In other words, in a given time instant more than one user is served at each beam in a multicast fashion [3]. The user signals are precoded at the Earth station and they are transmitted to the satellite through the feeder link. The satellite routes the received precoded signals through an array fed reflector able to radiate the multiuser information over a very large coverage area.

Apart from the well-known challenges in terrestrial multiantenna systems [2], multibeam satellite systems suffer from ad- ditional ones [1]. Among them, the high number of transmit antenna elements and beams limit the precoding designs due to optimization complexity. For instance, current Echostar 17 deploys 60 beams, requiring optimization of a complex vector of 3600 components.

Due to the challenges posed by the scale of the problem, current multibeam precoding schemes have been limited to closed-form expressions $[4,5]$ and semidefinite relaxation (SDR) based techniques over a reduced coverage area [6]. Similar to [6], the authors in [7] proposed the use of the feasible point pursuit successive convex approximation (FPP-SCA) [8] algorithm in order to decrease the computational complexity relative to [6]. Although FPP-SCA has lower worst-case computational complexity compared to the SDR approach [8], it might still not meet the requirements of next generation satellite systems where the number of beams are expected to increase.

Given this context, this paper proposes an optimization approach to further reduce the computational complexity of multibeam satellite precoding. This approach is based on posing the problem in a consensus optimization form, on which the alternating direction method of multipliers (ADMM) [9] is then applied. In contrast to FPP-SCA, which employs successive approximations, C-ADMM tackles the non-convex problem directly. Under certain conditions, it can be shown that the algorithm converges to a Karush-Kuhn-Tucker (KKT) point of this non-convex and NP-hard optimization problem. Our experience suggests that these conditions are fulfilled in our experiments. Furthermore, our results demonstrate that C-ADMM is able to provide a high quality, sub-optimal solution with a performance close to the solution obtained from FPP-SCA, yet at dramatically lower computational complexity.

\section{SYSTEM MODEL AND PROBLEM FORMULATION}

We consider a multibeam satellite system where the payload is equipped with $N$ feeds and serves a coverage area of $K$ beams. All beams share the frequency band and; in a given time instant, the $k$-th beam simultaneously serves $Q_{k}$ users. In other words, at a give time instant the scheduler selects a set of $Q_{k}$ users at the $k$-th beam (same for all beams) and it constructs a codeword with information to be transmitted to all $Q_{k}$. Without loss of generality, we assume that each beam serves the same number of users simultaneously and it is equal to $Q$, i.e., $Q_{k}=Q, \forall k \in\{1, \ldots, K\}$.

This work has received funding from the Spanish Ministry of Economy and Competitiveness (Ministerio de Economia y Competitividad) under project TEC2014-59255-C3-1-R (ELISA); and from the Catalan Government (2014SGR1567 and 2014SGR1551); and from the U.S. NSF, grant CIF-1525194. 
Under this context, the received signal can be modelled as

$$
\mathbf{y}=\mathbf{H x}+\mathbf{n},
$$

where $\mathbf{y} \in \mathbb{C}^{K Q \times 1}$ is a vector containing the received signals at each user terminal. The vector $\mathbf{n} \in \mathbb{C}^{K Q \times 1}$ contains the noise terms of each user terminal and we assume that they are Gaussian distributed with zero mean, unit variance and uncorrelated with both the desired signal and the other users noise terms (i.e. $E\left[\mathbf{n n}^{H}\right]=\mathbf{I}_{K Q}$ ). The channel matrix can be described as follows

$$
\mathbf{H}=\mathbf{A G} \text {, }
$$

where $\mathbf{A} \in \mathbb{R}^{K Q \times K Q}$ is diagonal matrix whose diagonal entries are the atmospheric fading terms corresponding to the $q$-th user in the $k$-th beam. Matrix $\mathbf{G} \in \mathbb{R}^{K Q \times N}$ takes into account the rest of the gain and loss factors. Its $(k q, n)$-th entry can be described as follows

$$
[G]_{k, n}=\frac{G_{R} a_{k q n}}{4 \pi \frac{d_{k q}}{\lambda} \sqrt{K_{B} T_{R} B_{W}}}
$$

where $d_{k q n}$ is the distance between the $q$-th user terminal in the $k$-th beam and the satellite, $\lambda$ is the carrier wavelength, $K_{B}$ is the Boltzmann constant, $B_{W}$ is the carrier bandwidth, $G_{R}^{2}$ the user terminal receive antenna gain, and $T_{R}$ the receiver noise temperature. The term $a_{k q n}$ refers to the gain from the $n$-th feed to the $q$-th user in the $k$-th beam. It is important to mention that the matrix $\mathbf{G}$ has been normalized to the receiver noise term.

We represent the channel matrix $\mathbf{H}$ in the following form

$$
\mathbf{H}=\left(\mathbf{h}_{1}, \ldots, \mathbf{h}_{K Q}\right)^{T},
$$

where $\mathbf{h}_{i} \in \mathbb{R}^{N \times 1}$ refers to the channel vector of the $i$-th user. For notational convenience the channel vector of the $q$-th user in the $k$ th beam is defined as $\mathbf{h}_{k, q}=\mathbf{h}_{k Q+q}$ for $k=1, \ldots, K$ and $q=$ $1, \ldots, Q$.

In order to minimize the multiuser interference generated by the full frequency reuse and the on-board beamforming generation, precoding is considered. Under this context, the transmitted symbol vector is given by (5), where $\mathbf{s} \in \mathbb{C}^{K \times 1}$ is a vector that contains the transmitted symbols which we assume are uncorrelated with unit norm $\left(E\left[\mathbf{s s}^{H}\right]=\mathbf{I}\right)$, and matrix $\mathbf{W} \in \mathbb{C}^{N \times K}$ is the linear precoding matrix to be designed.

$$
\mathbf{x}=\mathbf{W} \mathbf{s} .
$$

For notational convenience, matrix $\mathbf{W}$ can be decomposed as follows

$$
\mathbf{W}=\left(\mathbf{w}_{1}, \ldots, \mathbf{w}_{K}\right),
$$

where $\mathbf{w}_{i}$ is the precoding vector for the $i$-th user.

Our precoder design criterion is to minimize the total transmitted power subject to minimum per-user SINR requirements and perantenna power constraints (PAPCs). Formally, the problem can be stated as

$$
\begin{aligned}
& \underset{\mathbf{w}_{1}, \ldots, \mathbf{w}_{K}}{\operatorname{minimize}} \sum_{k=1}^{K}\left\|\mathbf{w}_{k}\right\|^{2} \\
& \text { subject to } \\
& \quad \underset{q=1, \ldots, Q}{\operatorname{minimum}} \operatorname{SINR}_{k, q} \geq \gamma_{k}, \forall k \in\{1, \ldots, K\}, \\
& {\left[\sum_{k=1}^{K} \mathbf{w}_{k} \mathbf{w}_{k}^{H}\right]_{n n} \leq P, \forall n \in\{1, \ldots, N\}}
\end{aligned}
$$

where

$$
\operatorname{SINR}_{k, q}=\frac{\left|\mathbf{h}_{k, q}^{H} \mathbf{w}_{k}\right|^{2}}{\sum_{j \neq k}^{K}\left|\mathbf{h}_{k, q}^{H} \mathbf{w}_{j}\right|^{2}+1} .
$$

$\gamma_{k}$ is the target SINR of the $k$-th user and $P$ is the maximum per-feed transmit power. The constraints in (7) can be expressed as

$$
\begin{aligned}
& \underset{\mathbf{v}}{\operatorname{minimize}}\|\mathbf{v}\|_{2}^{2} \\
& \text { subject to } \\
& \qquad \mathbf{v}^{H} \mathbf{A}_{k, q} \mathbf{v}+\gamma_{k} \leq 0, \forall k \in\{1, \ldots, K\}, \forall q \in\{1, \ldots, Q\}, \\
& \quad \mathbf{v}^{H} \mathbf{B}_{n} \mathbf{v}-P \leq 0, \forall n \in\{1, \ldots, N\}
\end{aligned}
$$

where

$$
\mathbf{v}=\left(\mathbf{w}_{1}^{T}, \ldots, \mathbf{w}_{K}^{T}\right)^{T},
$$

$$
\begin{gathered}
\mathbf{A}_{k, q}=\gamma_{k} \sum_{j \neq k}^{K}\left(\mathbf{I}_{K N}+\mathbf{J}_{j} \otimes \mathbf{h}_{k, q} \mathbf{h}_{k, q}^{H}\right)-\mathbf{J}_{k} \otimes \mathbf{h}_{k, q} \mathbf{h}_{k, q}^{H}, \\
\mathbf{B}_{n}=\mathbf{I}_{K} \otimes \mathbf{M}_{n},
\end{gathered}
$$

where $\mathbf{I}_{K N}$ is a $K N$-dimensional identity matrix, $\mathbf{J}_{j}$ is a $K \times K$ zero matrix whose $j$-th diagonal entry is equal to 1 . In addition, $\mathbf{e}_{j}$ is a $N$-dimensional vector with all entries equal to 0 apart from the $j$-th entry which is set to one. Finally, $\mathbf{M}_{n}$ is a $N \times N$ zero matrix whose $n$-th diagonal entry is equal to 1 and $\otimes$ denotes the Kronecker product.

From (9), it can be observed that the set of SINR constraints might be non-convex since $\mathbf{A}_{k, q}$ can be indefinite for certain $k$ and $q$. In order to solve this problem, the system designer can opt to employ the FPP-SCA method described in [8]. This scheme approximates the original problem via a sequence of convex optimization problems of the following form

$$
\underset{\mathbf{v}, \mathbf{s}}{\operatorname{minimize}}\|\mathbf{v}\|_{2}^{2}+\psi\|\mathbf{s}\|_{1}
$$

subject to

$$
\begin{aligned}
& \mathbf{v}^{H} \mathbf{A}_{k, q}^{(+)} \mathbf{v}+2 \operatorname{Re}\left\{\mathbf{v}^{(t), H} \mathbf{A}_{k, q}^{(-)} \mathbf{v}\right\}-\mathbf{v}^{H} \mathbf{A}_{k, q}^{(-)} \mathbf{v}+\gamma_{k} \leq[\mathbf{s}]_{(k-1) Q+q}, \\
& \forall k \in\{1, \ldots, K\}, \forall q \in\{1, \ldots, Q\} \\
& \mathbf{v}^{H} \mathbf{B}_{n} \mathbf{v}-P \leq[\mathbf{s}]_{k}, \forall n \in\{1, \ldots, N\}, \forall k \in\{K Q+1, \ldots, K Q+N\} \\
& {[\mathbf{s}]_{k} \geq 0, \forall k \in\{1, \ldots, K Q+N\}}
\end{aligned}
$$

where $\mathbf{v}^{(t)}$ is the current iterate, $\mathbf{s}$ is the vector of slack variables, and

$$
\begin{gathered}
\mathbf{A}_{k, q}^{(+)}=\gamma_{k} \sum_{j \neq k}^{K}\left(\mathbf{I}_{K N}+\mathbf{J}_{j} \otimes \mathbf{h}_{k, q} \mathbf{h}_{k, q}^{H}\right), \\
\mathbf{A}_{k, q}^{(-)}=\mathbf{J}_{k} \otimes \mathbf{h}_{k, q} \mathbf{h}_{k, q}^{H} .
\end{gathered}
$$

Now, the optimization problem in (13) is convex as the non-convex quadratic functions have been replaced by their corresponding convex restrictions. The slack variables ensure problem feasibility at every step, and we impose an $\ell_{1}$-penalty on the slacks to ensure that they are used sparingly. The algorithm requires a randomly generated point $\mathbf{v}^{(0)}$ for initialization and proceeds by iteratively solving (13) and then setting the solution to be the next iterate. This results in a monotonically non-increasing cost function and once feasibility is attained, (i.e., $\mathbf{s}=\mathbf{0}$ ), it suffices to remove the slack variables from (13) and continue the successive approximation process. In this second stage of the algorithm, under certain regularity conditions, every limit point of the generated iterates is a KKT point of (9) 
[10]. At each step of FPP-SCA, we require to solve a convex problem of the form (13), which can be posed as a Second-order cone programming (SoCP) problem, and has substantially lower theoretical worst-case complexity relative to SDR based approaches [8,7]. Furthermore, the SDR techniques require a randomization algorithm coupled with a power control problem to generate feasible solutions for (9). Given these complexity considerations, we omit SDR from comparison in our simulations and limit ourselves to FPP-SCA as a performance benchmark. The stopping criteria for FPP-SCA was set to be $\left|\left\|\mathbf{v}^{(t)}\right\|^{2}-\left\|\mathbf{v}^{(t-1)}\right\|^{2}\right| \leq \epsilon_{\mathrm{FPP}-\mathrm{SCA}}$.

\section{CONSENSUS-ADMM}

As discussed in [9], Consensus-ADMM (C-ADMM) can be applied on the original problem (9) directly. Using variable splitting, CADMM decomposes (9) into smaller, low complexity sub-problems with simple, parallelizable updates. As a first step towards applying this algorithm, we express (9) in the following consensus form.

$$
\begin{aligned}
& \underset{\mathbf{v},\left\{\mathbf{z}_{l}\right\}_{l=1}^{L}}{\operatorname{minimize}}\|\mathbf{v}\|_{2}^{2} \\
& \text { subject to } \\
& \qquad \begin{aligned}
\mathbf{z}_{l}^{H} \mathbf{A}_{k, q} \mathbf{z}_{l} \leq \gamma_{l}, \forall l=(k-1) Q+q, \\
\\
\qquad k \in\{1, \ldots, K\}, \forall q \in\{1, \ldots, Q\}
\end{aligned} \\
& \quad \mathbf{z}_{l}^{H} \mathbf{B}_{l} \mathbf{z}_{l} \leq P, \forall l \in\{K Q+1, \ldots, L\} \\
& \quad \mathbf{v}=\mathbf{z}_{l}, \forall l \in\{1, \ldots, L\}
\end{aligned}
$$

where $L=K Q+N$ and the consensus variables $\left\{\mathbf{z}_{l}\right\}_{l=1}^{L}$ serve as local copies of the global variable $\mathbf{v}$. The algorithm takes the form of the following updates

$$
\begin{aligned}
& \mathbf{v} \leftarrow \frac{\rho}{1+L \rho}\left(\sum_{l=1}^{L}\left(\mathbf{z}_{l}+\mathbf{u}_{l}\right)\right) \\
& \mathbf{z}_{l} \leftarrow \underset{\mathbf{z}_{l}}{\operatorname{minimize}}\left\|\mathbf{z}_{l}-\mathbf{v}+\mathbf{u}_{l}\right\|_{2}^{2} \\
& \text { subject to } \\
& \mathbf{z}_{l}^{H} \mathbf{A}_{k, q} \mathbf{z}_{l} \leq-\gamma_{l}, \quad l=k Q+q, \quad \text { iff } \quad 1 \leq l \leq K Q, \\
& \quad \mathbf{z}_{l}^{H} \mathbf{B}_{l-K Q} \mathbf{z}_{l} \leq P, \quad \text { iff } \quad K Q+1 \leq l \leq L \\
& \mathbf{u}_{l} \leftarrow \mathbf{u}_{l}+\mathbf{z}_{l}-\mathbf{v}
\end{aligned}
$$

where $\mathbf{u}_{l}$ are the scaled dual variables of $l$-th constraint in (16). We point out that sub-problem (17b) can always be optimally solved (irrespective of (non)-convexity) as it is a QCQP problem with a single constraint. The form of the updates (17) allows the operations (17b) and (17c) to be implemented locally in $L$ distributed processors via global broadcast of $\mathbf{v}$ to all processors, while the update of the global variable (17a) is performed by gathering all the local updates $\left\{\mathbf{z}_{l}, \mathbf{u}_{l}\right\}_{l=1}^{L}$.

The parameter $\rho$ effects a trade-off between enforcing consensus among the local variables $\left\{\mathbf{z}_{l}\right\}_{l=1}^{L}$ and decreasing the cost function; for high values of $\rho$, the algorithm focuses on finding a consensus solution while for low values it favors the reduction of the cost function. The range of possible values of $\rho$ is problem dependent. According to [9] whenever a feasible initial point is used, $\rho$ can take low values leading to a large reduction of the required iterations. A feasible point can be obtained with the proposed C-ADMM technique via changing operation (17a) by

$$
\mathbf{v} \leftarrow \frac{1}{L}\left(\sum_{l=1}^{L}\left(\mathbf{z}_{l}+\mathbf{u}_{l}\right)\right) .
$$

Bearing this in mind, the proposed C-ADMM method has two phases. First, a feasible point is obtained via the operation in (17) but with the $\mathbf{v}$ update (18). The second phase consists of the operation (17) given a small value $\rho$. Furthermore, if for a chosen $\rho$, consensus amongst the local variables is achieved and the sequence of the global variables generated is convergent, then any limit point of the sequence satisfies the KKT conditions of (9) [9, Appendix A].

We now describe the particulars of solving the QCQP problem (17b) at each iteration of C-ADMM. According to [9], the optimization problem has a trivial solution $\mathbf{z}_{l}^{*}=\boldsymbol{\xi}_{l}=\mathbf{v}-\mathbf{u}_{l}$ if $\boldsymbol{\xi}_{l}$ does not violate the constraint in (17b); otherwise, it can be reduced to finding a zero of the following scalar function

$$
\Phi_{l}\left(\mu_{l}\right)=\sum_{i=1}^{K} N \frac{\lambda_{i}^{l}}{\left(1+\mu_{l} \lambda_{i}^{l}\right)}\left|\left[\boldsymbol{\xi}_{l}\right]_{i}\right|^{2}-c_{l},
$$

where $\lambda_{i}^{l}$ is the $i$-th eigenvalue of matrix

$$
\mathbf{A}_{k, q} \quad \text { iff } \quad 1 \leq l \leq K Q, \quad l=k Q+q
$$

or

$$
\mathbf{B}_{l} \quad \text { iff } \quad K Q+1 \leq l \leq K Q+N .
$$

Similarly, $c_{l}$ takes values either $-\gamma_{l}$ or $P$ depending on the index $l$. The optimal solution $\mu_{l}^{*}$ can be obtained by solving (19) via bisection. Finally, $\mathbf{z}_{l}^{*}$ is obtained via

$$
\mathbf{z}_{l}^{*}=\mathbf{Q}_{l}\left(\mathbf{I}+\mu_{l}^{*} \mathbf{L}_{l}\right)^{-1} \mathbf{Q}_{l}^{H} \boldsymbol{\xi}_{l},
$$

where $\mathbf{L}_{l}$ is a diagonal matrix that contains the eigenvalues and $\mathbf{Q}_{l}$ the eigenvectors of either matrices (20) or (21) depending on $l$.

We note that a total of $K Q+N$ consensus variables have to be stored in memory at each iteration, which can prove to be very expensive, especially when large coverages areas are targeted. In order to alleviate this problem, we propose to modify the C-ADMM algorithm by allocating a single consensus variable $\mathbf{z}_{K Q+1}$ to the entire set of PAPCs $(K Q+1 \leq l \leq L)$, instead of allocating one consensus variable to each of the $N$ PAPCs. The resulting sub-problem associated with this update now becomes

$$
\begin{aligned}
& \underset{\mathbf{z}_{K Q+1}}{\operatorname{minimize}}\left\|\mathbf{z}_{K Q+1}-\mathbf{v}+\mathbf{u}_{K Q+1}\right\|_{2}^{2} \\
& \text { subject to } \\
& \quad \mathbf{z}_{K Q+1}^{H} \mathbf{B}_{l-K Q} \mathbf{z}_{K Q+1} \leq P, \quad K Q+1 \leq l \leq N .
\end{aligned}
$$

The key aspect of this update is that it admits a closed form solution given by the Euclidean projection of $\boldsymbol{\xi}_{K Q+1}$ onto the constraint set of (23), which can be succinctly expressed as

$$
\mathbf{z}_{K Q+1}^{*}=\boldsymbol{\Pi}_{\mathcal{F}}\left(\boldsymbol{\xi}_{K Q+1}\right),
$$

where $\Pi_{\mathcal{F}}(\cdot)$ denotes the Euclidean projection onto the convex set

$$
\mathcal{F}: \mathbf{z}_{K Q+1}^{H} \mathbf{B}_{l-K Q} \mathbf{z}_{K Q+1} \leq P, \quad K Q+1 \leq l \leq N .
$$

Consequently, the number of consensus variables is reduced from $K Q+N$ to $K Q+1$. We note that while our proposed modification decreases overall complexity, it does not come at the expense of the simplicity of the updates. The overall algorithm is depicted in 
Algorithm 1.

\section{SIMULATION RESULTS}

In order to evaluate the performance of the proposed algorithm, we consider a real coverage area of a geostationary satellite system. This data has been obtained in a study performed by the European space agency (ESA). We assume that at each time instant all bandwidth is shared by all beams and $Q$ users per beam and frame is served. The system parameters are not detailed due to space limitations. The reader can refer to [1] for further details. In all simulations we have performed 400 Monte-Carlo runs with a Windows desktop with 4 Intel i5 cores and $4 \mathrm{~GB}$ of RAM.

Data: $\mathbf{H},\left\{\gamma_{k}\right\}_{k=1}^{K}, P$.

1 Initialize $\mathbf{v},\left\{\mathbf{z}_{l}\right\}_{l=1}^{K+1},\left\{\mathbf{u}_{l}\right\}_{l=1}^{K+1}$;

2 Compute the eigen-decomposition of $\mathbf{A}_{k}=\mathbf{Q}_{k} \mathbf{L}_{k} \mathbf{Q}_{k}$ for $k=1, \ldots, K$;

3 while $\mathbf{v}$ is not feasible do

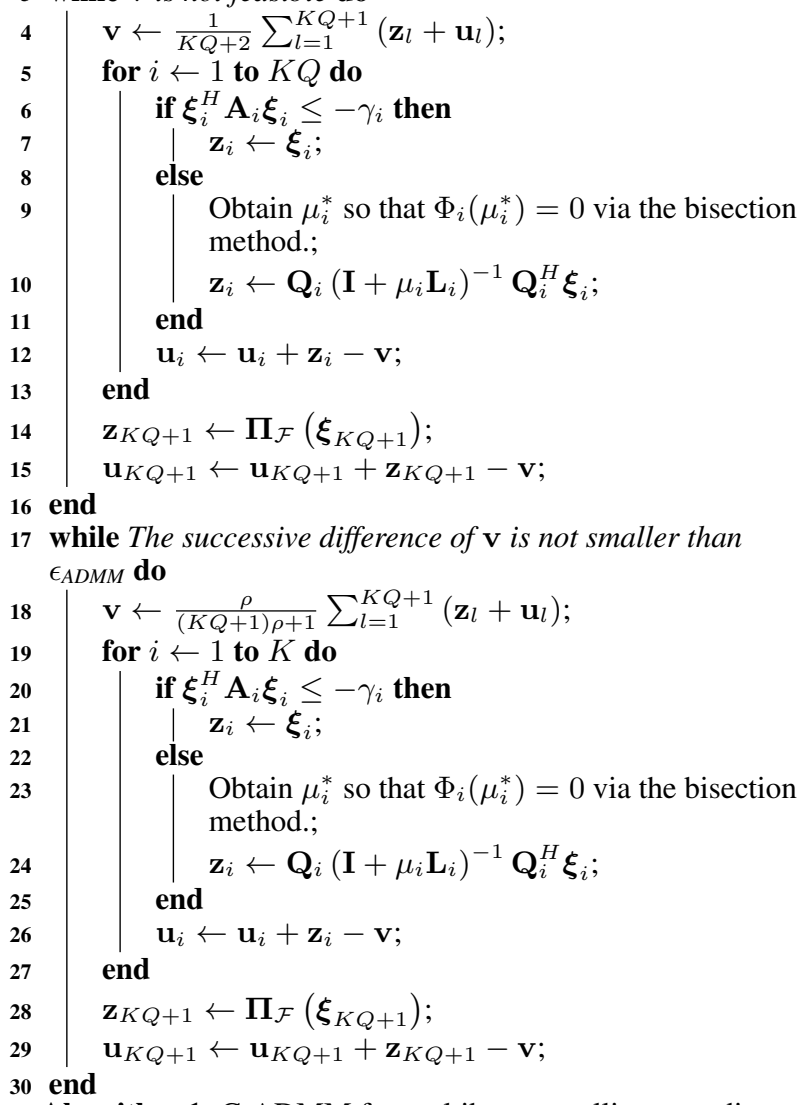

Algorithm 1: C-ADMM for multibeam satellite precoding

Figure 1 depicts the average computational time of FPP-SCA and C-ADMM for different values of $Q$ maintaining $N=K=4$. In both cases, $\epsilon_{\mathrm{FPP}-\mathrm{SCA}}=\epsilon_{\mathrm{ADMM}}=10^{-4}$. For the C-ADMM scheme we set $\rho=25$ and for FPP-SCA we set $\psi=1$. We set a maximum iteration counter of $10^{4}$ iterations for C-ADMM. In case the method does not converge for this number of iterations, we use a new random initial point. Moreover, the maximum available power per feed is set to 55 Watts and $\gamma_{k}=\gamma=0 \mathrm{~dB}, \forall k \in\{1, \ldots, K\}$. The figure reveals that both FPP-SCA and C-ADMM lead to very similar average transmit power for all values of $Q$. However, it is also evident that for all values of $Q, \mathrm{C}-\mathrm{ADMM}$ requires much lesser computational

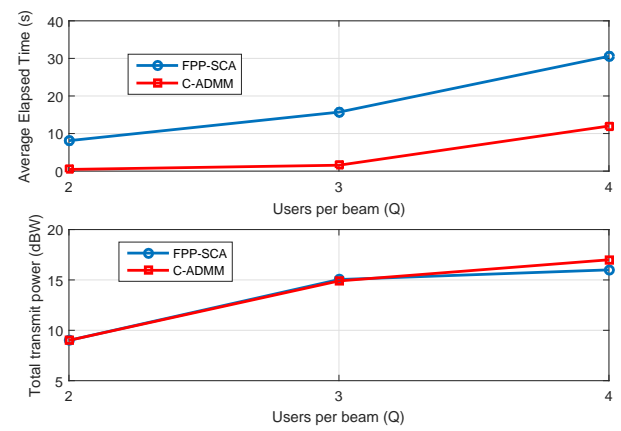

Fig. 1: Average computational time (up) and total transmit power (down) of FPP-SCA and C-ADMM for different values of $Q$.

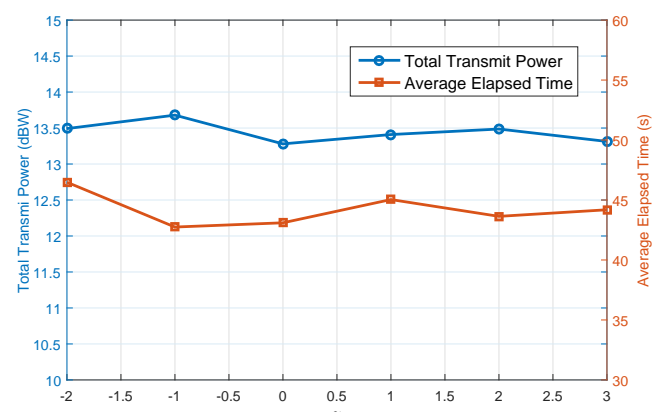

Fig. 2: Average computational time and transmit power for CADMM

time compared to FPP-SCA (upto 3-fold improvement for large $Q$ ). In this experiment, C-ADMM converged in all realizations.

The results of another experiment are depicted in Figure 2, which shows the average computational time of a system with $N=K=8$ and $Q=2$ for different values of minimum $\operatorname{SINR}(\gamma)$ and with a maximum per feed transmit power of 55 Watts. Impressively, C-ADMM is able to keep the average computational time low even for this large coverage area. It is important to note that the computational time and average transmit power are very similar for all values of $\gamma$. In contrast to the previous setting, C-ADMM does not converge for certain realizations, so re-initialization was required in this case, which resulted to an average of 1.3 trials per realization over all values of $\gamma$.

\section{CONCLUSIONS}

In this paper, we cast the multibeam satellite precoder design optimization problem in the form of multigroup multicasting with PAPCs. The C-ADMM algorithm was used to obtain high quality, sub-optimal solutions for this non-convex and NP-Hard optimization problem. C-ADMM directly tackles the problem by first reformulating it in consensus optimization form, which results in QCQP sub-problems with a single constraint. These problems can always be solved to global optimality in an efficient manner, even when they lack convexity. We proposed a modification to C-ADMM which reduces overall complexity in our context while preserving the simplicity of the updates. Numerical simulations reveal that the proposed scheme exhibits lower computational complexity and distributed implementation capabilities when compared to the state-of-the-art, while providing very similar performance results. 


\section{REFERENCES}

[1] M. Á. Vázquez, A. I. Pérez-Neira, D. Christopoulos, S. Chatzinotas, B. Ottersten, P. D. M. Arapoglou, A. Ginesi, and G. Taricco, "Precoding in Multibeam Satellite Communications: Present and Future Challenges," to appear in IEEE Wireless Communications Magazine, 2016.

[2] E. Karipidis, N. D. Sidiropoulos, and Z. Q. Luo, "Quality of Service and Max-Min Fair Transmit Beamforming to Multiple Cochannel Multicast Groups," IEEE Transactions on Signal Processing, vol. 56, no. 3, pp. 1268-1279, March 2008.

[3] Pantelis-Daniel M. Arapoglou, Alberto Ginesi, Stefano Cioni, Stefan Erl, Federico Clazzer, Stefano Andrenacci, and Alessandro Vanelli-Coralli, "DVB-S2X-enabled precoding for high throughput satellite systems," Int. J. Satellite Communications Networking, vol. 34, no. 3, pp. 439-455, 2016.

[4] Giorgio Taricco, "Linear Precoding Methods for Multi-Beam Broadband Satellite Systems," in European Wireless 2014; 20th European Wireless Conference; Proceedings of, May 2014, pp. 1-6.

[5] Jesus Arnau, Bertrand Devillers, Carlos Mosquera, and Ana Perez-Neira, "Performance study of multiuser interference mitigation schemes for hybrid broadband multibeam satellite architectures," EURASIP Journal on Wireless Communications and Networking, vol. 2012, no. 1, pp. 132, 2012.

[6] D. Christopoulos, S. Chatzinotas, and B. Ottersten, "Multicast Multigroup Precoding and User Scheduling for Frame-Based Satellite Communications," Wireless Communications, IEEE Transactions on, vol. PP, no. 99, pp. 1-1, 2015.

[7] D. Christopoulos, S. Chatzinotas, and B. Ottersten, "Multicast multigroup beamforming for per-antenna power constrained large-scale arrays," in 2015 IEEE 16th International Workshop on Signal Processing Advances in Wireless Communications (SPAWC), June 2015, pp. 271-275.

[8] O. Mehanna, K. Huang, B. Gopalakrishnan, A. Konar, and N. D. Sidiropoulos, "Feasible Point Pursuit and Successive Approximation of Non-Convex QCQPs," IEEE Signal Processing Letters, vol. 22, no. 7, pp. 804-808, July 2015.

[9] K. Huang and N. D. Sidiropoulos, "Consensus-ADMM for General Quadratically Constrained Quadratic Programming," IEEE Transactions on Signal Processing, vol. 64, no. 20, pp. 5297-5310, Oct 2016.

[10] Amir Beck, Aharon Ben-Tal, and Luba Tetruashvili, "A sequential parametric convex approximation method with applications to nonconvex truss topology design problems," Journal of Global Optimization, vol. 47, no. 1, pp. 29-51, 2010. 